\title{
Synthetic applications of chiral 1,3-dioxolan-4-ones and 3-acyloxazolidin-5-ones
}

\author{
R. Alan Aitken,* Steven D. McGill, and Lynn A. Power
}

School of Chemistry, University of St Andrews, North Haugh, St Andrews, Fife, KY16 9ST, U. K. E-mail: raa@st-and.ac.uk

\begin{abstract}
Chiral dioxolanones derived from $\alpha$-hydroxy acids can act as chiral acyl anion equivalents for addition to ethyl crotonate, nitroalkenes and butenolide. Subsequent flash vacuum pyrolysis results in loss of $\mathrm{CO}$ and $\mathrm{Bu}^{\mathrm{t}} \mathrm{CHO}$ to give products. The related $N$-acyl and $N$-thioacyl-1,3oxazolidin-5-ones formed from $\alpha$-amino acids behave in a quite different way with loss of $\mathrm{CO}_{2}$ upon pyrolysis leading via $N$-(thio)acylaziridines to oxazolines and thiazolines. The stereochemistry of these transformations, both relative and absolute, is discussed and a mechanism involving an azomethine carboxylate intermediate is proposed.
\end{abstract}

Keywords: Dioxolanones, acyl-anion, oxazolidiones, acylaziridines, oxazolines, thiazolines

\section{Contents}

1. Introduction

2. Results and Discussion

3. Acknowledgements

4. References

\section{Introduction}

Some time ago we reported that 1,3-dioxolan-4-ones readily formed from $\alpha$-hydroxy acids could act as acyl anion equivalents in a sequence involving deprotonation, alkylation and flash vacuum pyrolysis (FVP) to generate carbonyl products (Scheme 1$){ }^{1}$ 


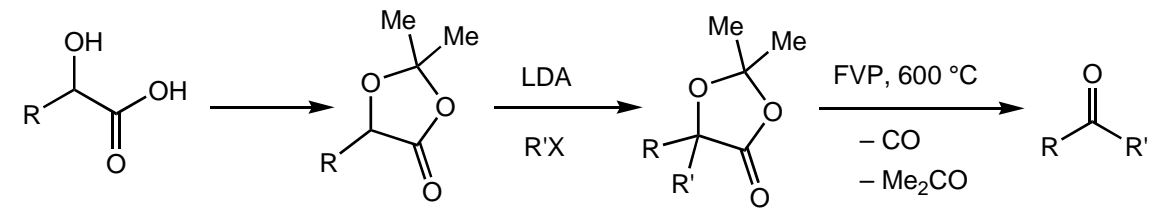

\section{Scheme 1}

This was extended to provide chiral acyl anion equivalents by using the dioxolanones derived from $S$-mandelic or lactic acid and pivalaldehyde and carrying out their addition to ethyl crotonate (Scheme 2). ${ }^{1}$

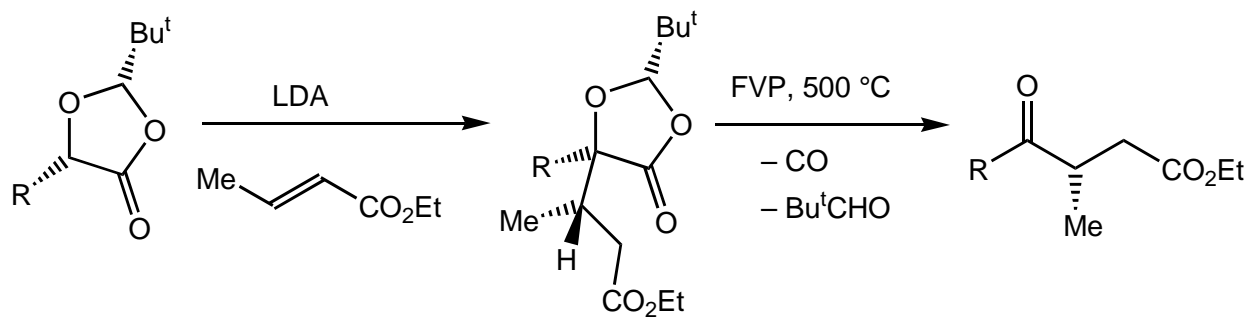

\section{Scheme 2}

$\mathrm{R}=\mathrm{Ph}$ 33\% e.e. $\mathrm{R}=$ Me $86 \%$ e.e.

We now present further developments of this chemistry as well as that of the related 1,3oxazolidin-5-ones which behave in a completely different way upon pyrolysis.

\section{Results and Discussion}

The first area we have addressed is to widen the range of available R groups by using $\alpha$-hydroxy acids derived from diazotisation of $\alpha$-amino acids (Scheme 3 ). While the procedure to form the dioxolanones is straightforward, pure pivalaldehyde is rather expensive [100 g = $£ 183$ (€ 280)].

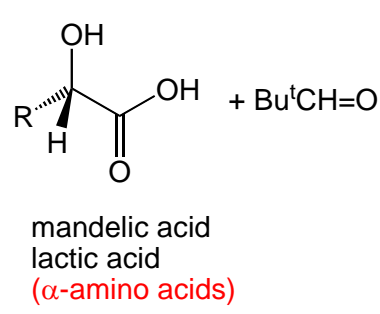

lactic acid
( $\alpha$-amino acids)

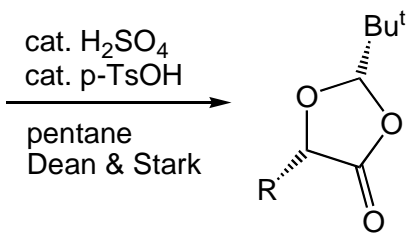

$\mathrm{R}=\mathrm{Ph}, \mathrm{Me}$

$\mathrm{R}=\mathrm{Pr}^{\mathrm{i}}, \mathrm{CH}_{2} \mathrm{Ph}, \mathrm{Bu}^{\mathrm{i}}, \mathrm{Bu}^{\mathrm{s}}$ etc.

\section{Scheme 3}

Several attempts were made to solve this problem, including preparation by reaction of the tert-butyl Grignard reagent with methyl formate on a 1 mole scale, ${ }^{2}$ and transfer hydrogenation 
of pivaloyl chloride using formic acid and ammonia. ${ }^{3}$ Neither of these methods were entirely satisfactory. Finally a source of pivalaldehyde as a $75 \%$ solution in tert-butanol at a much more reasonable price $[100 \mathrm{~g}=£ 31$ (€ 45)] was located. We were pleased to find that this works fine for the dioxolanone formation shown above. Particularly for the compounds derived from $S$ valine and $S$-phenylalanine, large amounts of diastereomerically pure dioxolanones have been obtained for further study.

The range of electrophiles has also been widened to include $\beta$-nitrostyrenes and butenolide. The adducts formed from these as well as the expected final pyrolysis products are shown in Scheme 4.
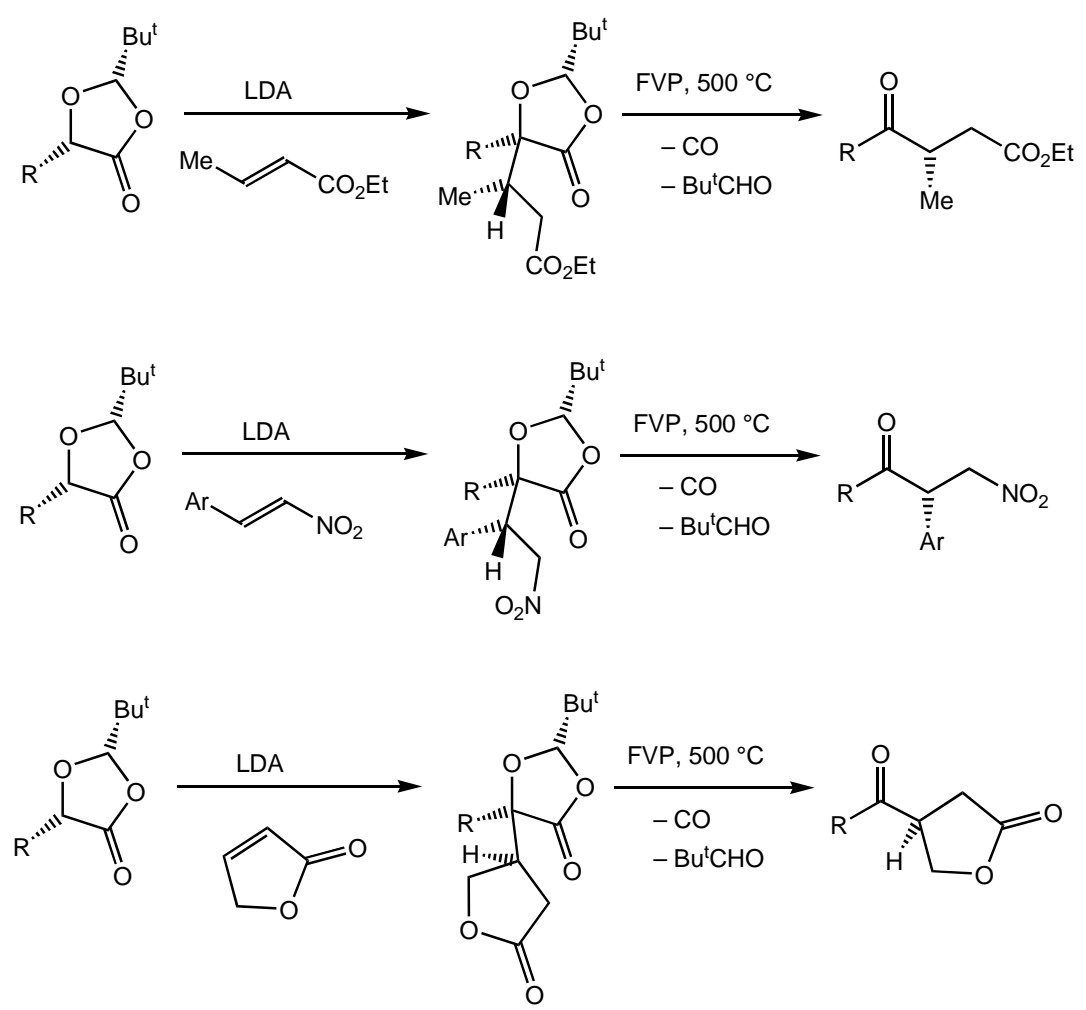

\section{Scheme 4}

Using the mandelic-acid derived dioxolanone with $p$-methoxy- $\beta$-nitrostyrene gave the two diastereomers shown in a fairly equal ratio (Scheme 5). This contrasts with the work of Pedro et al. who, by adding the dangerous carcinogen HMPA, were able to get high selectivity of 9:1 in favour of the opposite diastereomer. ${ }^{4}$ In fact we were not overly worried by this result since the two diastereomers were readily separable by simple column chromatography and thus both could be obtained in reasonable amount and in pure form. 


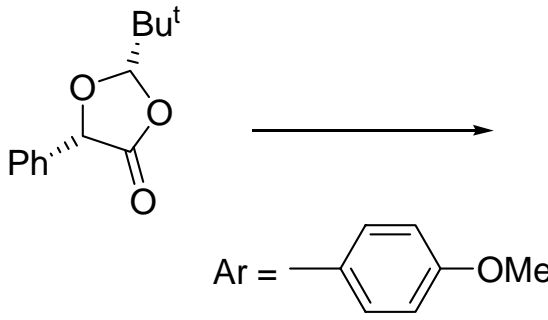<smiles>O=C1O[C@@H](Br)O[C@]1([AlH])[C@H]([AlH2])C[N+](=O)[O-]</smiles><smiles>O=C1O[C@@H](Br)O[C@]1([AlH])[C@H](Cl)C[N+](=O)[O-]</smiles>

cf. with added HMPA

1

9

\section{Scheme 5}

This fact allowed the absolute configuration to be determined with certainty for the first time by an X-ray diffraction study of the major isomer (Pedro's minor isomer) which gave the structure shown in Figure 1 with the $S, S, S$ configuration.
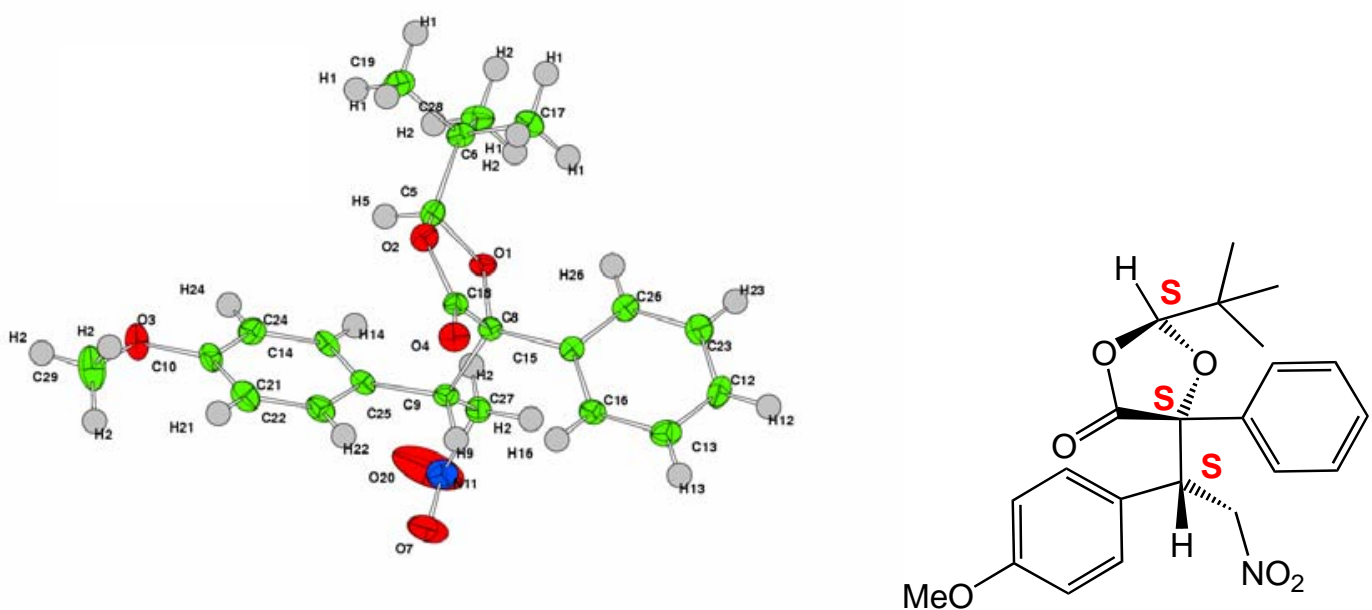

Figure 1

By considering the two transition states shown below (Scheme 6), it can be seen that the basis for selectivity is really not great and the ratio of 4:3 obtained is really not surprising.

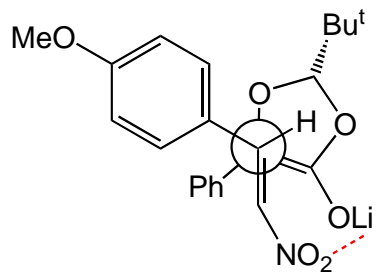

More favoured leads to major S,S,S product

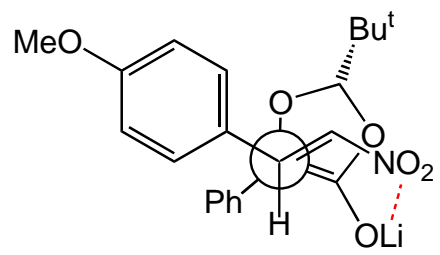

Less favoured (except with HMPA) leads to S,S,R product

\section{Scheme 6}


A preliminary attempt to carry out the final pyrolytic step gave a good yield of $p$ methoxystyrene but this is not too surprising since aliphatic nitro compounds are well known to be unstable under thermal conditions. The desired ketone probably is generated (Scheme 7) but it does not survive the conditions. We feel the solution to this is to reduce the nitro group to the amine (or a protected form thereof) prior to the pyrolysis step and this is currently being investigated.

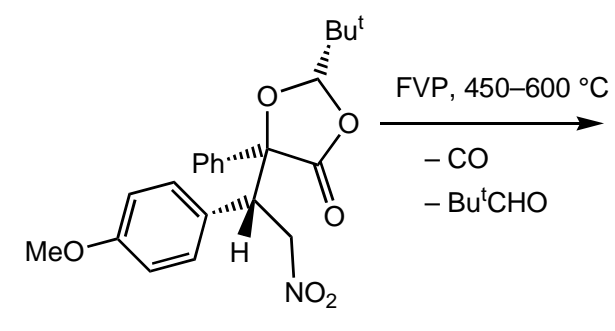<smiles>COc1ccc(C(C[N+](=O)[O-])C(=O)c2ccccc2)cc1</smiles>

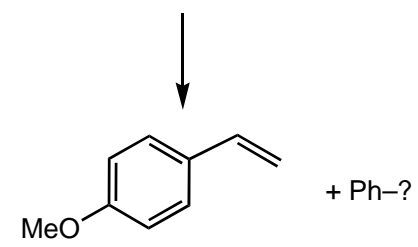

\section{Scheme 7}

A second major area of work involves the chiral $N$-acyl-1,3-oxazolidin-5-ones which can be obtained by treatment of the sodium salts of $\alpha$-amino acids with benzaldehyde followed by cyclisation of the resulting imine with an acyl chloride (Scheme 8). Treatment of the products with Lawesson's reagent readily gives the corresponding $N$-thioacyl compounds.
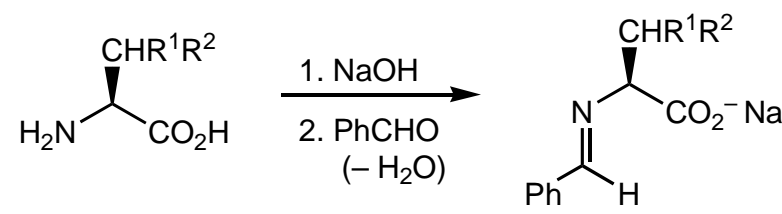

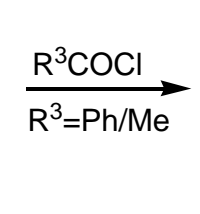<smiles>[R12]C[C@H]1C(=O)O[C@@H](c2ccccc2)N1C([R1])=O</smiles><smiles>[R2]C[C@H]1C(=O)O[C@@H](c2ccccc2)N1C([R])=S</smiles>

\section{Scheme 8}


Based on the precedent of the dioxolanones, it was expected that these might behave as shown in Scheme 9 and thus provide alternative acyl anion equivalents.<smiles></smiles><smiles>[R]C[C@H]1C(=O)O[C@@H](c2ccccc2)N1C([R])=O</smiles>

\section{Scheme 9}

To allow the thermal behaviour of these systems to be examined for the first time, a good range of compounds have been prepared and fully characterised (Scheme 10). In most cases the spectra are complicated by the occurrence of restricted rotation of the $N$-acyl or thioacyl groups leading to broad spectra and double peaks. In some cases this has been quantified and for the first compound shown in Scheme 10, a variable temperature NMR study gave a barrier to rotation around $\mathrm{N}-\mathrm{COMe}$ of $63.8 \mathrm{~kJ} \mathrm{~mol}^{-1}$ and an energy difference between forms of $2.5 \mathrm{~kJ} \mathrm{~mol}^{-1}$.<smiles>CC(=O)N1[C@H](c2ccccc2)OC(=O)[C@@H]1[C@@H]1C(=O)O[C@@H](c2ccccc2)N1C(C)=O</smiles><smiles>CCCC[C@@H]1C(=O)O[C@@H](c2ccccc2)N1C(=O)N1[C@H]([C@H]2C(=O)O[C@@H](c3ccccc3)N2C(=O)c2ccccc2)OC(=O)[C@H]1c1ccccc1</smiles><smiles>CC(=S)N1[C@H](c2ccccc2)OC(=O)[C@H]1C</smiles><smiles>CC(=S)N1[C@@H](Cc2ccccc2)C(=O)O[C@H]1c1ccccc1</smiles><smiles>C[C@H]1C(=O)O[C@@H](c2ccccc2)N1C(=S)c1ccccc1</smiles><smiles>C[C@H]1C(=O)O[C@@H](c2ccccc2)N1C(=S)c1ccccc1</smiles><smiles>CCC[C@@H]1C(=O)O[C@@H](c2ccccc2)N1C(=S)c1ccccc1</smiles><smiles>O=C1O[C@@H](c2ccccc2)N(C(=S)c2ccccc2)[C@@H]1Br</smiles>

\section{Scheme 10}

An X-ray structure of this compound also confirmed the trans relative configuration (Figure 2). 


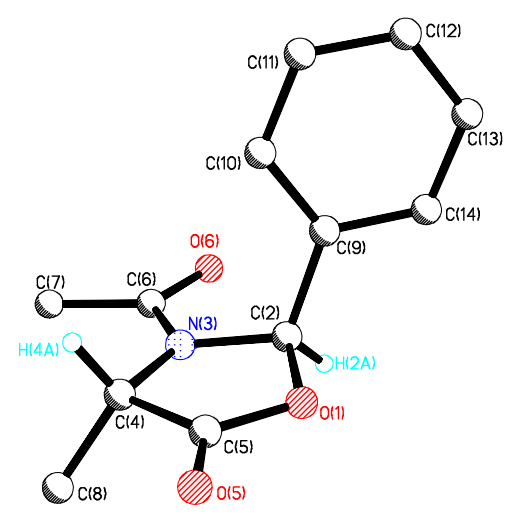

Figure 2

The pyrolytic study of these compounds has given some very interesting results which are still not fully understood. The basic observation is that, rather than loss of $\mathrm{CO}$, there is initial loss of $\mathrm{CO}_{2}$ at $\mathrm{FVP}$ temperatures as low as $450{ }^{\circ} \mathrm{C}$ to give the aziridines and at higher temperatures of $550{ }^{\circ} \mathrm{C}$ these undergo the known ring-expansion to 2-oxazolines. For most of the compounds the relative stereochemistry of these transformations is as follows:<smiles>[R2]C[C@H]1C(=O)O[C@@H]([R14]([H])([H])[H])N1C([R])=O</smiles>

However for the compounds derived from phenylglycine this is now different:

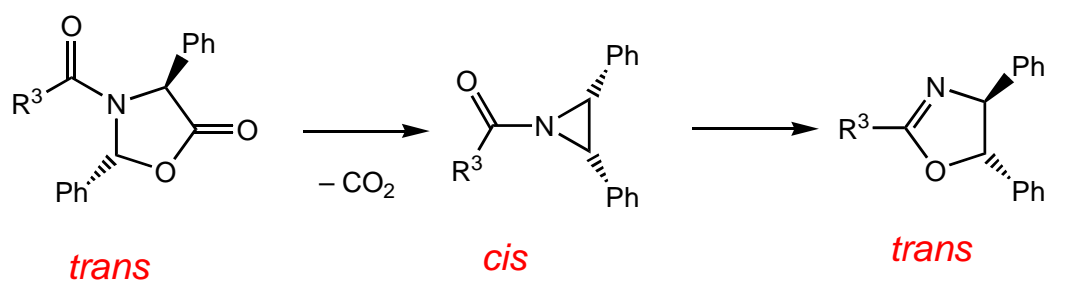

For the thioacyl compounds the basic chemistry is similar but the aziridines are now too reactive to be isolated and go straight through to the thiazolines. 


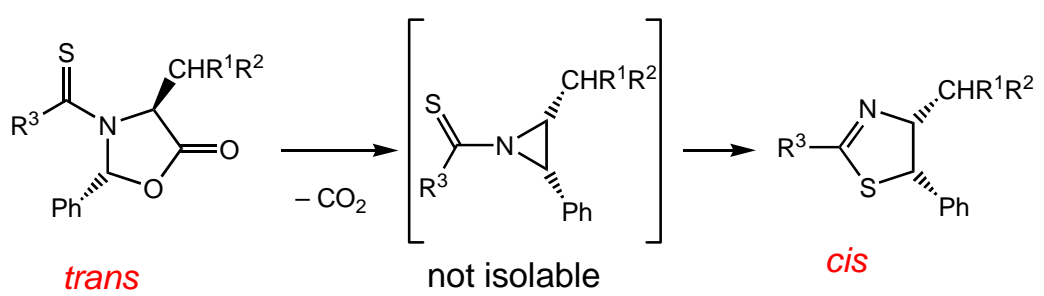

These reactions of such oxazolidinones, appear to be previously unknown but some precedent can be found in similar systems such as the oxazolidine-2,4-diones, ${ }^{5}$ oxazolin-5-ones, ${ }^{6}$ and oxathiolan-5-ones. ${ }^{7,8}$

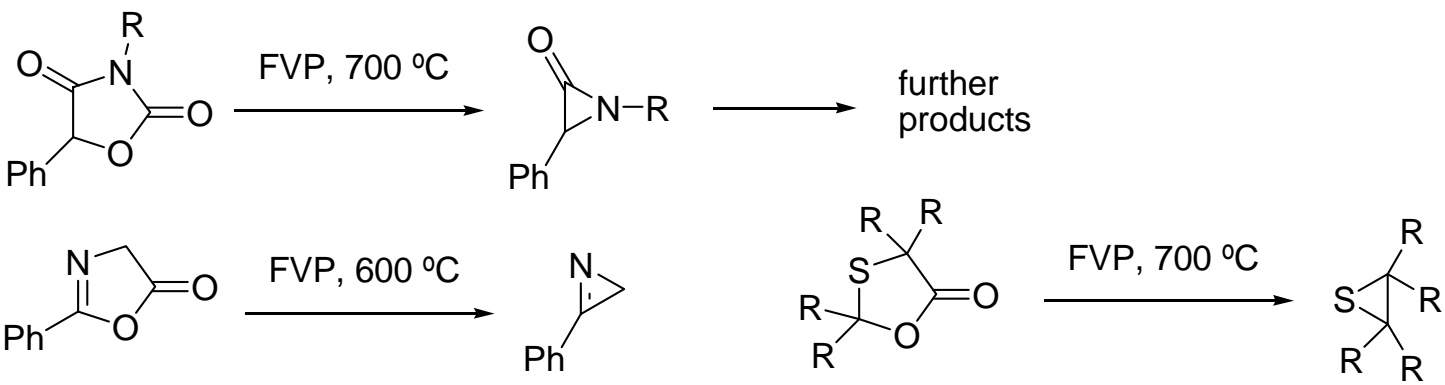

What is somewhat surprising about our pyrolysis results, and still not fully understood, is that all the products appear to be non-racemic - in other words there has been some retention of absolute stereochemistry. While the second, ring-expansion step is well known and known to be stereoselective, it is the initial extrusion of $\mathrm{CO}_{2}$ where retention of absolute stereochemistry is somewhat unexpected. We propose that the most likely explanation is involvement of a chiral azomethine carboxylate intermediate where the ring-closure is already well advanced before the $\mathrm{CO}_{2}$ has fully departed as shown in Scheme 11 .

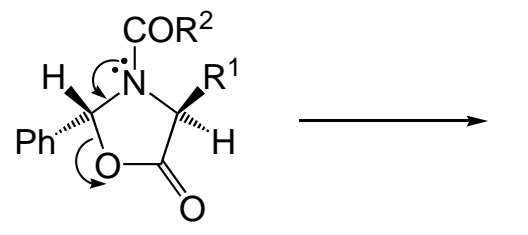

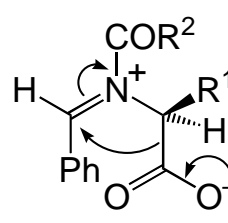<smiles>[R]C(=O)N1C([R1])C1Pc1ccccc1</smiles>

\section{Scheme 11}

In fact, it may be the possibility of such an intermediate that favours extrusion of $\mathrm{CO}_{2}$ in the first place, as also happens for the same reason with the oxathiolanones of Cameron and Pinnick. It is only in the dioxolanone case when such an intermediate would be much less stable that the fragmentation is instead diverted toward loss of CO.

Further aspects of these studies as well as full experimental details will be reported shortly. 


\section{Acknowledgements}

We thank Professor Alexandra M. Z. Slawin, University of St Andrews, for determining the Xray crystal structures.

\section{References}

1. Aitken, R. A.; Thomas, A. W. Synlett 1998, 102.

2. Campbell, K. N. J. Am. Chem. Soc. 1937, 59, 1980.

3. Shamsuddin, K. M.; Zobairi, M. O.; Musharraf, M. A. Tetrahedron Lett. 1998, 39, 8153.

4. Blay, G.; Fernández, I.; Monje, B.; Pedro, J. R. Tetrahedron 2004, 60, 165.

5. $\quad$ Aitken, R. A.; Thomas, A. W. Arkivoc 2002, 3 (iii), 71.

6. Lesniak, S.; Mloston, G.; Heimgartner, H. Pol. J. Chem. 1998, 72, 1915.Cameron, T. B.; Pinnick, H. W. J. Am. Chem. Soc. 1979, 101, 4755.

8. Cameron, T. B.; Pinnick, H. W. J. Am. Chem. Soc. 1980, 102, 744. 